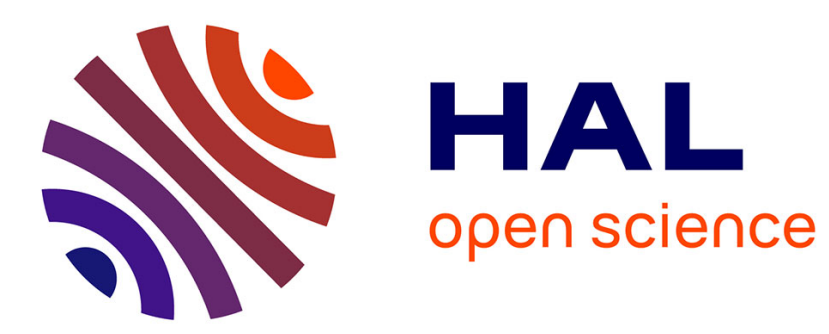

\title{
Transition to turbulence through steep global-modes cascade in an open rotating cavity
}

\author{
B. Viaud, Eric Serre, Jean-Marc Chomaz
}

\section{To cite this version:}

B. Viaud, Eric Serre, Jean-Marc Chomaz. Transition to turbulence through steep globalmodes cascade in an open rotating cavity. Journal of Fluid Mechanics, 2011, 688, pp.493-506. 10.1017/jfm.2011.393 . hal-00998000

\section{HAL Id: hal-00998000}

https://hal-polytechnique.archives-ouvertes.fr/hal-00998000

Submitted on 8 Jul 2014

HAL is a multi-disciplinary open access archive for the deposit and dissemination of scientific research documents, whether they are published or not. The documents may come from teaching and research institutions in France or abroad, or from public or private research centers.
L'archive ouverte pluridisciplinaire HAL, est destinée au dépôt et à la diffusion de documents scientifiques de niveau recherche, publiés ou non, émanant des établissements d'enseignement et de recherche français ou étrangers, des laboratoires publics ou privés. 


\title{
Transition to turbulence through steep global-modes cascade in an open rotating cavity
}

\author{
Bertrand Viaud $^{1,2} \dagger$, Eric Serre ${ }^{2}$ and Jean-Marc Chomaz \\ ${ }^{1}$ Centre de Recherche de l'Armée de l'Air, CReA BA701 13661 Salon de Provence, France \\ ${ }^{2}$ M2P2, CNRS Universités Aix Marseille, IMT Chateau-Gombert 13451 Marseille, France \\ ${ }^{3}$ LadHyX, CNRS-Ecole Polytechnique, F-91128 Palaiseau, France
}

(Received 12 July 2011; revised 12 July 2011; accepted 11 September 2011;

first published online 28 October 2011)

The transition to turbulence in a rotating boundary layer is analysed via direct numerical simulation (DNS) in an annular cavity made of two parallel corotating discs of finite radial extent, with a forced inflow at the hub and free outflow at the rim. In a former numerical investigation (Viaud, Serre \& Chomaz J. Fluid Mech., vol. 598, 2008, pp. 451-464) realized in a sectorial cavity of azimuthal extent $2 \pi / 68$, we have established the existence of a primary bifurcation to nonlinear global mode with angular phase velocity and radial envelope coherent with the so-called elephant mode theory. The former study has demonstrated the subcritical nature of this primary bifurcation with a base flow that keeps being linearly stable for all Reynolds numbers studied. The present work investigates the stability of this elephant mode by extending the cavity both in the radial and azimuthal direction. When the Reynolds number based on the forced throughflow is increased above a threshold value for the existence of the nonlinear global mode, a large-amplitude impulsive perturbation gives rise to a self-sustained saturated wave with characteristics identical to the 68 -fold global elephant mode obtained in the smaller cavity. This saturated wave is itself globally unstable and a second front appears in the lee of the primary where small-scale instability develops. These secondary instabilities are identical for the $2 \pi / 68$ and the $2 \pi / 4$ long sectorial cavities, indicating that transition involves a Floquet mode of zero azimuthal wavenumber. This secondary instability leads to a very disorganized state, defining the transition to turbulence. The observed transition to turbulence linked to the secondary instability of a global mode confirms, for the first time on a real flow, the possibility of a direct transition to turbulence through an elephant mode cascade, a scenario that was up to now only observed on the Ginzburg-Landau model.

Key words: absolute/convective instability, boundary layer stability, rotating flows

\section{Introduction}

The flow above an infinite rotating disc is an example of three-dimensional boundary layers where cross-flow instability can develop as over swept wings. When a second rotating disc is added parallel to the first, the configuration schematizes the cavity between the discs holding the blades of a turbine or compressor. For corotating discs

$†$ Email address for correspondence: bertrand.viaud@supaero.org 
of finite extent with a forced inflow, early theoretical and experimental results were obtained by Hide (1968). The steady flow may be decomposed into four regions: an inflow and an outflow zone framing a solid-body rotating core sandwiched by two thin Ekman boundary layers. Later work by Owen \& Pincombe (1980) has confirmed these results for the stationary flow and added measurements of Ekman-layer instabilities. A review of the subject can be found in Owen \& Rogers (1995). The recent numerical work by Viaud, Serre \& Chomaz (2008) (from now on labelled VSC) has demonstrated the similarity of the local velocity profiles in the boundary layers over a single rotating disc and in an annular rotating cavity with a forced throughflow. For the cavity, VSC have shown that the flow is linearly stable but nonlinearly unstable with large initial perturbation giving rise to self-sustained nonlinear global mode with a steep front located at the transition point between convective and absolute instability (the so-called elephant mode). Transposed to the case of the disc this finding of a subcritical global instability conciliates both the linear results of Davies \& Carpenter (2003) and the nonlinear dynamics proposed by Pier (2003).

In the parallel-flow approximation, linear stability analysis has revealed that the flow above the single infinite rotating disc is subject to two generic types of instability. An inviscid instability, due to the inflexional nature of the velocity profile, is labelled type I, whereas type II is due to the combined action of viscous and Coriolis effects (see the review by Crespo del Arco et al. 2005). The type I inflexional instability undergoes a transition from convective to absolute instability at a Reynolds number just below the value found for transition to turbulence in the experiments (Lingwood 1995, 1996, 1997). However, the non-parallel numerical study of Davies \& Carpenter (2003) has shown that the flow over a single disc is linearly globally stable, the stabilization being attributed to the detuning: the radial variation of absolute frequency (Davies, Thomas \& Carpenter 2007). Following the approach of Chomaz, Huerre \& Redekopp (1991) these authors devised a selection criterion for global frequency, while taking into account a radial variation of the local absolute frequency. In the case of the rotating-disc flow, when using a non-local time scale, the temporal frequency of the absolute instability varies linearly with the radius. This linear variation of the temporal absolute frequency will promote linear global stability whenever it is stronger than the corresponding variation of the local absolute growth rate.

However, nonlinearities can counterbalance this stabilizing effect when they reach a sufficient magnitude and lead to a nonlinear global mode, even in the case of linear global stability (Chomaz 1992). In the strongly nonlinear and weakly nonparallel regime, the presence of an absolutely unstable region of finite extent is a sufficient condition for the existence of a nonlinear global mode with a steep front, located at the upstream border of the absolute region, separating upstream evanescent perturbations from a downstream saturated wave. This so-called elephant mode was first described in Tobias, Proctor \& Knobloch (1998) and Pier et al. (1998). Couairon \& Chomaz (1999) have shown, on a model equation, that when the saturated primary wave behind the front of the global mode is itself absolutely unstable to local secondary perturbations, direct transition to disorder can occur. Pier (2003) has demonstrated that the saturated type I wave which should follow the primary front on a rotating disc if an elephant mode were present is already itself absolutely unstable. The primary nonlinear global mode on a rotating disc should therefore be unstable toward a secondary instability global mode with the possibility of a rapid transition to turbulence shortly behind the primary front. One should emphasize that on the single rotating disc the existence of a nonlinear global mode, be it stable or unstable to secondary perturbations has not yet been established numerically or analytically. More 


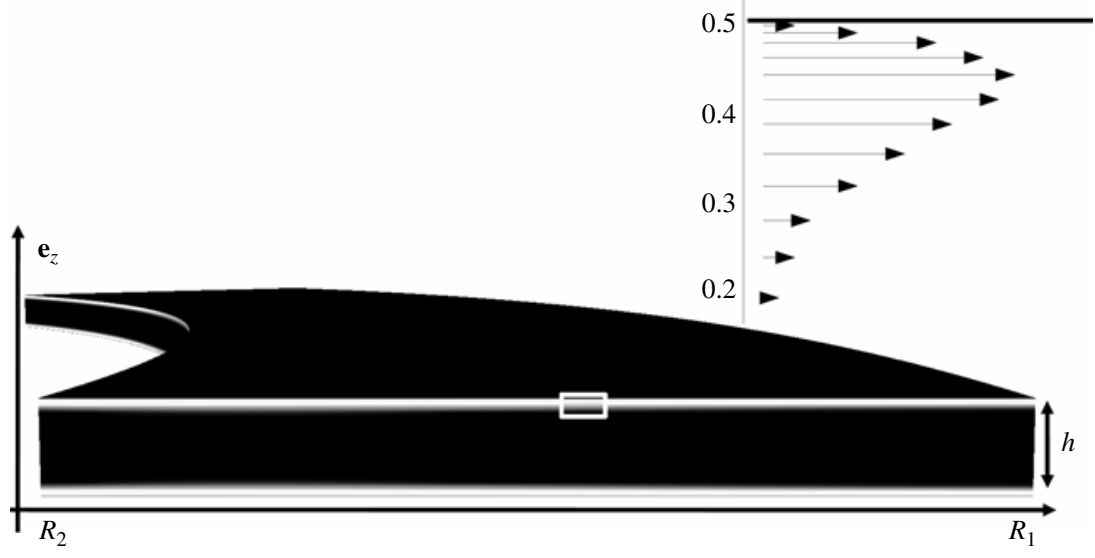

FIGURE 1. Sketch of the annular cavity. Shades of grey in the $(r, z)$-plane map the radial velocity field of the base flow. Centrifugal Ekman layers appear in white and the radial velocity vanishes in black areas. The vector field is a close-up of the upper Ekman layer in the white box.

generally, to best of the authors' knowledge, the direct route to turbulence through a cascade of absolute instabilities (initially proposed by Huerre 1988 as a generic shortcut on the route to turbulence) has been observed only on a model equation (Couairon \& Chomaz 1999) but never identified on a numerical simulation of a real flow. The present work is therefore the first observation of this direct cascade scenario on a real flow. In a previous paper on the $2 \pi / 68$ rotating cavity with throughflow, VSC have observed the existence of a subcritical global bifurcation toward an elephant mode of azimuthal wavenumber 68 . The present paper addresses the question of the secondary stability of the resulting global mode by relaxing the $2 \pi / 68$ periodicity constrain previously imposed and by running the same computation in a cavity twice as long in the radial direction and 17 times wider domain in the azimuthal direction. Identical computations have been performed both in a cavity of angular extent $2 \pi / 68$ and $2 \pi / 4$, to discriminate the effect of the domain size in the radial direction and of the periodicity. Longer runs have been performed using a standard Large eddy simulation (LES) method to approach statistical stationarity of the flow.

The configuration is presented in $\S 2$. Section 3 introduces the numerical method, with particular stress on key points. Results of numerical runs are discussed in $\S 4$ and exhibit a global behaviour with two successive fronts leading to a disordered domain. The possible origin of this spatial transition to turbulence is further discussed in $\S 5$.

\section{Configuration}

The configuration (figure 1), similar to that presented by VSC, is made of two corotating discs of finite radial extent, having same axis and angular velocity $\left(\boldsymbol{\Omega}_{d}^{*}=\Omega_{d}^{*} \mathbf{e}_{z}\right)$ and separated by a gap of height $h^{*}$. The cavity is open at both inner and outer radius $R_{1}^{*}$ and $R_{2}^{*}=R_{1}^{*}+\Delta R^{*}$, and a flow is forced at the hub and exits at the rim. The geometry is defined by two control parameters, the curvature $R_{m}=\left(R_{2}^{*}+R_{1}^{*}\right) / \Delta R^{*}$ and the aspect ratio $L=\Delta R^{*} / h^{*}$. For the long cavity of this paper, the values $L=10$ and $R_{m}=5$ have been retained, to be compared with $L=5$ and $R_{m}=9$ in VSC.

The volume flow rate $Q^{*}$ is imposed at the hub through the use of Dirichlet boundary conditions on the velocity, using a precomputed balanced profile (see 
VSC for details on the balanced profile computation). The flow is defined by two global control parameters, the throughflow parameter $C_{w}=Q^{*} /\left(\nu R_{1}^{*}\right)$ and the Reynolds number $\operatorname{Re}_{h}=\left(h^{*} / \delta^{*}\right)^{2}=h^{* 2} \Omega_{d}^{*} / v$ which represents the ratio of the cavity height $\left(h^{*}\right)$ and the Ekman length scale $\left(\delta^{*}=\sqrt{\nu / \Omega_{d}^{*}}\right)$. The Navier-Stokes equations written in the frame rotating at $\Omega_{d}^{*}$ are made non-dimensional using the time, length, velocity and pressure scales $t_{\text {ref }}=\Omega_{d}^{*^{-1}}, l_{\text {ref }}=h^{*}, V_{\text {ref }}=\Omega_{d}^{*} h^{*}$ and $P_{\text {ref }}=(1 / 2) \rho^{*} \Omega_{d}^{*^{2}} R_{1}^{*^{2}}$, respectively.

When $C_{w} R e_{h}^{-1 / 2}$ is small, Ekman's linearity assumption holds for the stationary axisymmetric solution, with nonlinear effects negligible compared with the Coriolis force. An asymptotic solution for the flow in the cavity can then be obtained by matching Ekman solutions for two single infinite discs (Zandbergen \& Dijkstra 1986; Serre et al. 2001):

$$
\begin{array}{r}
u(r, z)=-V_{g}(r)\left[\exp \left(-\frac{z+\frac{1}{2}}{\delta}\right) \sin \left(\frac{z+\frac{1}{2}}{\delta}\right)-\exp \left(\frac{z-\frac{1}{2}}{\delta}\right) \sin \left(\frac{z-\frac{1}{2}}{\delta}\right)\right] \\
v(r, z)=V_{g}(r)\left[1-\exp \left(-\frac{z+\frac{1}{2}}{\delta}\right) \cos \left(\frac{z+\frac{1}{2}}{\delta}\right)-\exp \left(\frac{z-\frac{1}{2}}{\delta}\right) \cos \left(\frac{z-\frac{1}{2}}{\delta}\right)\right]
\end{array}
$$

with $(u, v)$ the radial and azimuthal velocity in the frame rotating with the discs, $\delta=R e_{h}^{-1 / 2}$ the non-dimensional Ekman layer thickness and $V_{g}$ the geostrophic velocity

$$
V_{g}(r) / r=-C_{w} R e_{h}^{-1 / 2} R_{1} /\left(2 \pi r^{2}\right)
$$

which is small in the asymptotic limit. For the base-flow asymptotic solution the throughflow occurs in the Ekman layers only, forcing the core of the fluid to rotate at a slightly smaller differential rotation rate $V_{g}(r) / r$, compared with the disc rotation rate $\Omega_{d}=1$. Note that axial velocity in the core $w(r, z)$ scales as $\delta V_{g}(r) / r$ and is asymptotically smaller than $u$ or $v$. Local stability properties are controlled by local parameters, the Rossby number $(R o)$ and the local Reynolds number $\left(R e_{\delta}\right)$

$$
R o(r)=\frac{V_{g}(r)}{r} \quad \operatorname{Re}_{\delta}(r)=\left|V_{g}(r)\right| \delta e_{h}=\left|V_{g}(r)\right| R e_{h}^{1 / 2} .
$$

For the base-flow asymptotic solution (2.1) analytical expressions of these local parameters can be derived using (2.3): $R e_{\delta}^{a}=R_{1} C_{w} /(2 \pi r)$ and $R o^{a}=-\operatorname{Re}_{\delta} /\left(r \sqrt{R e_{h}}\right)$. In the direct numerical simulation (DNS) $C_{w} R e_{h}^{-1 / 2}$ is finite and the asymptotic solution is not valid. The velocity $V_{g}(r)$ is then not given by (2.3) but directly measured in the midplane of the cavity. The present double-disc cavity differs from the single infinite rotating disc since for the cavity the flow rate is constant and the differential rotation between the fluid core and the disc decreases with $r$, whereas for the single disc the flow rate increases with $r$ and the differential rotation is constant. For the single-disc case, the local Reynolds number increases outward with $r$, and the Rossby number is a global control parameter independent of $r$. In the cavity configuration, the Reynolds number decreases with $1 / r$ and the Rossby number is a local parameter that decreases with $1 / r^{2}$. The critical Reynolds numbers $\left(\operatorname{Re}_{\delta}^{c}\right)$ associated with the different thresholds (convective instability, absolute instability) are then functions of the local Rossby number and are found to decrease faster than the actual local Reynolds number $\left(R e_{\delta}\right)$. Owing to the radial variation of the Rossby number, transition from convective to absolute instability may arise at a position where the local Rossby number is significantly different from the value of -1 used in most of the single-disc studies. 


\section{Numerics}

The pseudospectral collocation-Tchebyshev Fourier-Galerkin code has been described in VSC. When the flow becomes turbulent and a long computation has to be carried out in order to obtain converged results, calculations have been stabilized using the spectral vanishing viscosity (SVV) techniques (Severac \& Serre 2007). A new diffusion operator $\Delta_{S V V}$ is simply implemented by combining the classical diffusion and the new SVV terms to obtain

$$
v \Delta_{S V V} \equiv v \Delta+\nabla \cdot\left(\varepsilon_{N} Q_{N} \nabla\right)=v \nabla \cdot S_{N} \nabla
$$

where $v$ is the diffusive coefficient and where

$$
S_{N}=\operatorname{diag}\left\{S_{N_{i}}^{i}\right\}, \quad S_{N_{i}}^{i}=1+\frac{\varepsilon_{N_{i}}^{i}}{v} Q_{N_{i}}^{i}
$$

with $\varepsilon_{N_{i}}^{i}$ the maximum of viscosity and $Q_{N_{i}}^{i}$ a one-dimensional viscosity kernel operator acting in direction $i$, and defined in the spectral space by an exponential function: $\widehat{Q}_{N_{i}}^{i}(\omega)=0$, if $0 \leqslant \omega \leqslant \omega_{T}^{i}$ and $\widehat{Q}_{N_{i}}^{i}(\omega)=\exp \left(-\left(\omega-\omega_{N}^{i}\right)^{2} /\left(\omega-\omega_{T}^{i}\right)^{2}\right)$ if $\omega_{T}^{i} \leqslant \omega \leqslant \omega_{N}^{i}$, where $\omega_{T}^{i}$ is the threshold after which the viscosity is applied and $\omega_{N}^{i}$ the highest frequency calculated in the direction $i$. In present computations $\omega_{T}^{i}=10 \sqrt{N_{i}}$ and $\varepsilon_{N_{i}}^{i}=2 / N_{i}$. We note that the SVV operator affects at most two-thirds of the spectrum on the highest frequencies $\left(w_{T}=0\right)$ and, consequently, that DNS results are recovered for laminar flows.

Owing to the decomposition on a Fourier basis, it is straightforward to impose the chosen periodicity by concentrating the mesh points in a sector of angular extent $2 \pi / 68$ or $2 \pi / 4$.

To speed up computation, symmetry with respect to the midplane $(z=0)$ has been imposed and simulations ran in upper-half cavities. This reduction of the computational domain has been extensively validated in the shorter cavities (VSC), by comparing with full-height cavity simulations. Usual no-slip boundary conditions are applied at the disc and stress-free conditions at the lower plane, enforcing the symmetry with respect to the middle of the cavity. At the outflow, convective boundary conditions as in VSC were used in order to avoid reflections of the wave packets. At the inflow, precomputed velocity profiles, picked up from the computations of the axisymmetric flow in a shifted cavity overlapping the present one, were imposed. The very slight off-balance in the imposed velocity profiles brings about convectively unstable axisymmetric type II rolls that must be damped to keep them from reaching a noticeable amplitude through exponential growth all along the cavity, eventually modifying the underlying base flow through nonlinear interaction. Being axisymmetric they can only be filtered using a frequency criteria since the axisymmetric base flow must remain free to evolve in time. To avoid the appearance of these spurious waves, a buffer zone $(\Delta r=2)$ is placed at the inlet, where oscillations of the zero azimuthal wavenumber are damped using the selective frequency damping (SFD), as proposed by Akervik et al. (2006). This damping is limited to azimuthal wavenumber zero and to the very upstream part of the computational domain, between $r=20$ and $r=22$. Being applied well ahead of the primary front located at $r=24$, it is not altering its dynamics, as verified by comparison with short cavity results presented in VSC where no damping was applied since the total amplification of the axisymmetric mode was small enough to keep being in the background noise.

The final size of the mesh used is $649 \times 10 \times 65$ in the radial, azimuthal and axial directions, respectively, in the $1 / 68$ cavity, and $649 \times 170 \times 65$ in the $1 / 4$ 
cavity. To ensure that the front dynamics was grid-independent we have first checked the convergence of the spectral coefficients spectra. Moreover, in the $1 / 68$ cavity identical simulations were carried out with twice as many mesh points in the azimuthal direction (20 instead of 10). Prior to saturation no differences could be detected, and beyond saturation the maximum difference in perturbation-velocity amplitude was less than $5 \%$.

The cut-off scale has been determined based on an estimation of the inertial-range extent and on the assumption that the primary spiralling vortices correspond to the largest structures in the turbulent cascade. These vortices have been extracted from the DNS flow field, and their wavelength as well as their velocity has been measured. A Reynolds number $(R e)$ was constructed from these two values, and the inertial range extent assumed to be of the order of $R e^{-3 / 4}$ as predicted by the Kolmogorov theory for developed, isotropic and homogeneous turbulence. The resolution of the mesh is approximately 10 and 6 Kolmogorov length scales in the radial and axial directions, respectively, meaning that the turbulent dissipation scale is not resolved in the present computations. We need a long cavity to properly resolve the global mode structure and cannot afford at the same time a mesh fine enough to resolve the turbulent cascade down to the dissipation scale. As a result, when transition to turbulence is triggered and a long integration time is needed, energy piles up at the cut-off scale leading eventually to the divergence of the solution due to the nearly-zero numerical diffusivity. LES using the SVV as a subgrid model has been carried out to model the effects of the unresolved cascade and dissipation range. Such a model is very attractive because it keeps the spectral convergence (Severac \& Serre 2007).

The total simulations time is very long since one has to wait for the transient $(\Delta t=2.5)$ during which the three-dimensional convective impulse response is seen to end, and then for the fronts to stabilize. For this reason the finest mesh is not used from the start, but mesh resolution is increased when the spectra show that spectral aliasing becomes noticeable. The initial mesh is the same as in VSC $(433 \times 33 \times 10)$, but for a twice-as-long cavity, with a time step equal to $5 \times 10^{-5}$, each step consuming $0.27 \mathrm{~s}$ of CPU time on the NEC SX-8 of IDRIS. Computations with this mesh can be carried up to $t=10.49$ where they blow up. The solution taken at $t=10.4$ is projected on the finest possible grid $(649 \times 65 \times 10)$ and the computation restarted. The time step is now equal to $2 \times 10^{-5}$, and each one costs $1.1 \mathrm{~s}$ of CPU time. Any attempt to use a finer grid leads to poor conditioning of the matrix for the radial-derivative operator. Using this mesh the simulation can be pushed up to $t=10.6$ before blowing up. Perturbation-energy profiles, as demonstrated in figure 4 , are obtained using this fine mesh. From $t=10.4$, alternatively, the computation can be continued using SVV as a LES approach, keeping the lower-resolution mesh. LES computations remain stable and are run up to $t=15.8$ when they are voluntarily stopped because of CPU-time limitation. All of this process has been done again from the start in a cavity of angular extent $2 \pi / 4$ with 170 points in the azimuthal direction, leading to the same behaviour and results, but with a cost of $21 \mathrm{~s}$ of CPU time per time step when using the finer mesh.

The DNS code is also used to get local stability properties and in particular the precise location of the transition to absolute instability and the absolute frequency $\omega_{0}$ and spatial growth rate $k_{0}$ there, following the method of Perret et al. (2006). This approach yielded results in full agreement with those extrapolated from the single-disc flow analysis by Lingwood (1997) for the position, slope and frequency of the primary front as already shown in VSC. 


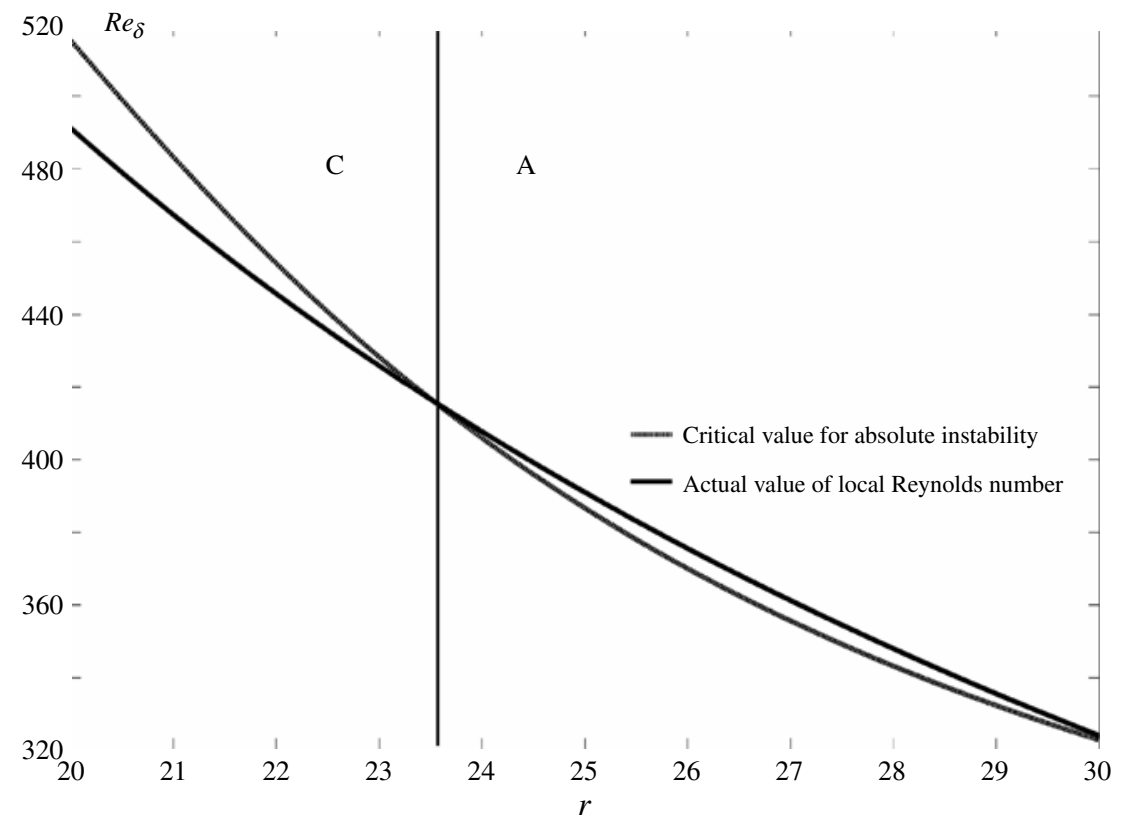

FIGURE 2. Predicted area of absolute instability in the computational domain, based on the radial distribution of the local Reynolds number (bold line) and the critical Reynolds number for absolute instability estimated through interpolation of Lingwood's data using the actual local value of the Rossby number which is varying with the radial position $r$.

\section{Results}

The global Reynolds number is set to $R e_{h}=780$, and the mass flow rate to $C_{w}=1995$ which places the transition from convective to absolute instability in the first half of the cavity and sufficiently downstream of the inlet buffer zone as shown in figure 2. Axisymmetric stationary base flow is first computed through the use of SFD extended to the entire cavity. As done in VSC the non-axisymmetric dynamics of the flow is analysed by superimposing a spatially localized perturbation during a single time step at the beginning of the computations. The perturbation velocity field corresponds to a Stokes flow over a hemispherical roughness of radius $R_{p} / h=0.008$ located at the wall near the hub. An $m$-fold azimuthal periodicity harmonics of the symmetry of the cavity may be imposed on the initial perturbation by placing in the $2 \pi / 4$ cavity $m / 4$ regularly spaced identical perturbations. This value for radius $R_{p} / h=0.008$ is the same as that used in VSC and as for the small cavity it triggers a saturated global mode in the long cavity. In the present cavity, where the end of the absolutely unstable domain is located at the downstream limit of the computational domain, the subcritical nature of the bifurcation is confirmed since the wave packet generated by a smaller initial perturbation with $R_{p} / h=0.002$ initially grows but eventually relaxes to zero. From now on only $R_{p} / h=0.008$ initial perturbation will be reported.

Figure 3 presents the nonlinear evolution of such an initial perturbation with a 68fold symmetry computed in the $1 / 68$ sectorial cavity. The spatiotemporal diagram on figure 3(a) is designed to show only the finite-amplitude part of the wave packet due to the thresholding effect of the grey-scale used. The initial impulse at time $t=0$ and $r=21$ is first too small to be visible. It saturates close to time $t=2$ where its leading 
edge keeps heading downstream until it reaches the outer boundary around $t=4$. The trailing edge moves upstream and rapidly reaches $r \simeq 24$ at $t=2$ before stabilizing progressively close to $r=23.5$ around $t=8$. Shortly after $t=3$, the saturated wave downstream of the trailing front starts being perturbed with order-unity perturbation close to $r=27$. This secondary perturbation moves up and then forms a stationary secondary front close to $r=25.5$ around $t=8$. Behind that secondary front the state is disordered in time and space indicating incipient turbulence. Beyond $t=8$ and up to $t=10.4$ the two fronts seem to stabilize. Shortly after $t=10.4$ the DNS blows up by pilling of energy at the truncation, visible when energy spectra are plotted, since the dissipation scale cannot be resolved. To allow the energy that cascades through nonlinear evolution of the primary and the secondary instabilities to be dissipated, LES simulations have been restarted from the DNS solution at time $t=10.4$. As seen on figure 3(a) after the discontinuity in the time axis marking the shift to the LES computation, the nearly steady state reached at $t=10.4$ is maintained up to $t=15.8$ where we stop the computation. During this phase the primary front keeps vacillating around the same mean position and seems nearly not affected by the use of the SVV whereas the secondary front very slightly moves upstream as a result of a change in the high radial and axial wavenumber dissipation.

The associated spatial structure is shown by horizontal cross-section of the axial velocity field taken at $t=8.4$, figure $3(b)$. The alternation of positive (white) and negative (black) axial velocity evidences spiralling vortices winding clockwise in space and rotating counterclockwise in time according to figure 3(a). The spiral saturates after the primary front at $r=23.5$, developing secondary instability close to $r=25.5$. Figure 3(c) shows the vertical cut of the axial velocity at the same instant, with the primary front visible as cross-flow rolls forming close to $r=23.5$. These vortices saturate and remain localized in the boundary layer before becoming unstable with perturbations invading the whole cavity depth close to $r=25.5$ (figures $3 c$ and 6). At this secondary front, axisymmetric flow modifications, measured by the azimuthal average of the axial velocity field, figure $3(d)$, become of order unity. Close to this secondary front the harmonic component $m=136$ also becomes of the same amplitude compared with the primary rolls as shown on figure 4 . The spatial onset of disorder is thus due to a small-scale instability but its physical origin is not easy to determine since the flow is directly disordered at the secondary front. Indeed a frequency decomposition of the flow done on the space-time diagram of figure 3(a) between time $t=4$ and $t=8$ when statistical steadiness is approximately achieved (figure 5) shows the bursting of all frequencies downstream of the secondary front at $r=25.5$, whereas the global mode frequency and its harmonics are experiencing saturation between the primary front close to $r=23.5$ and the secondary front close to $r=25.5$. The global mode frequency and its harmonics correspond to a Doppler effect of the rotating spiral which is stationary in a particular rotating frame. Spatial harmonics $136,204, \ldots$ of the base azimuthal wavenumber 68 have the same phase speed as the fundamental and are therefore associated to $2 \omega_{g}, 3 \omega_{g}, \ldots, \omega_{g}$ being the dominant frequency. The breaking of the temporal invariance (Gollub \& Swinney 1975) occurs only at secondary front and is presently associated with a direct transition to a broadband spectrum.

This secondary instability is noticeably different from that predicted by the stability analysis of Pier (2003) which should correspond to a subharmonic large-scale mode (seen on figure 10 in the cited paper) with $m=18$ for Rossby number unity. In the cavity the Rossby number is smaller than unity at the secondary front and the 

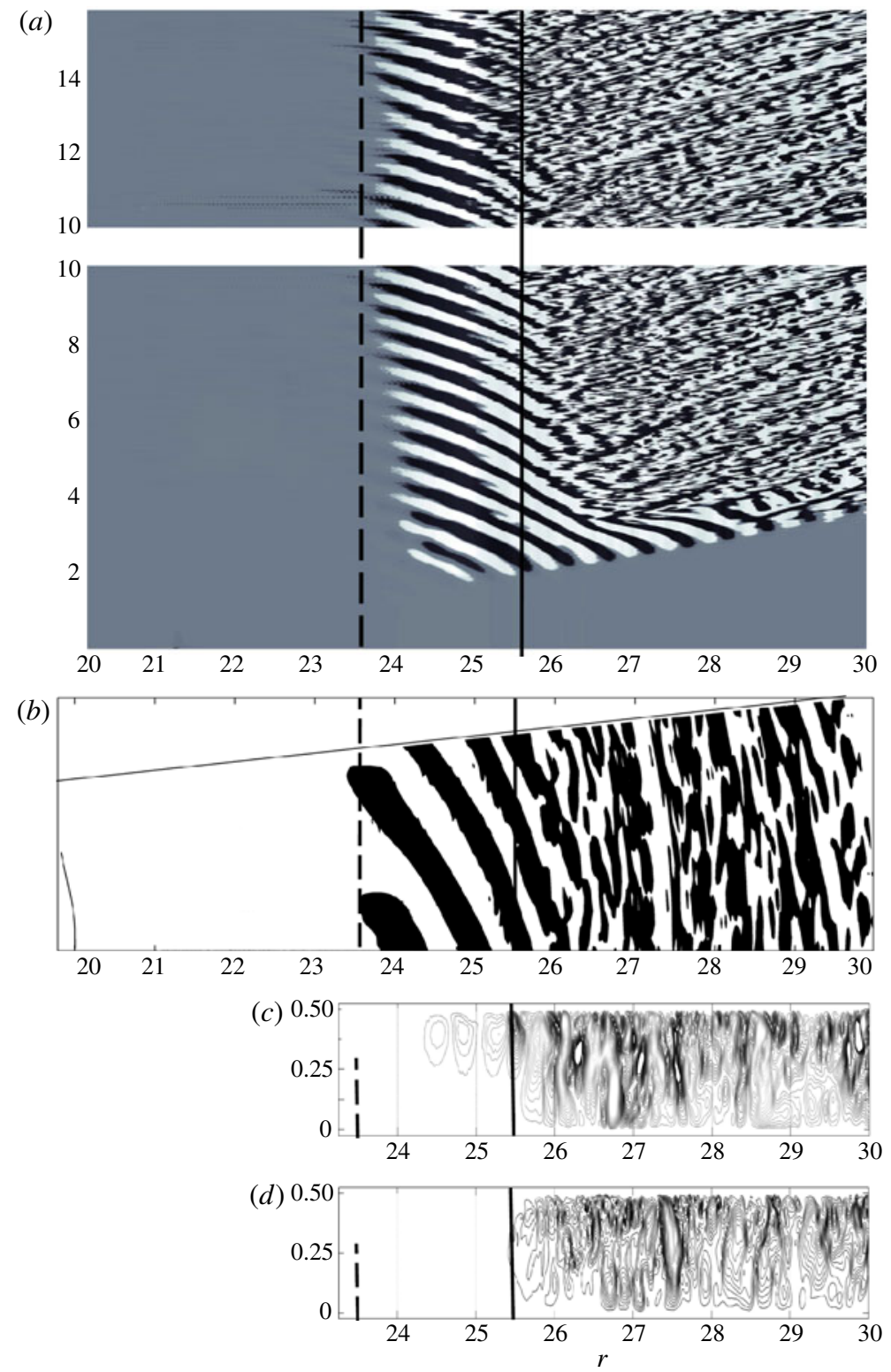

FIGURE 3. Nonlinear impulse response: (a) spatiotemporal recording of the axial velocity for given azimuth $\theta=0$ and height $z=0.49$ in the cavity: DNS results for $0<t<10.4$ and LES results for $10.4<t<15$.8. Grey scale saturates at $10^{-5}$. Globally unstable flow exhibiting a secondary front $(r \simeq 25.5)$ in the lee of the primary $(r \simeq 23.5)$. (b) Thresholding of axial velocity $\left(\left|v_{z}\right|>2 \times 10^{-5}\right)$ in an horizontal plane $z=0.49$ (time $t=8.4$ ), featuring the same fronts at $r=23.5$ and $r=25.5$, the change of radial wavelength from $\lambda=30 \delta$ to $\lambda=18 \delta$ corresponding to the saturation of the type I absolute mode is visible upstream the second front where disorder sets in. $(c)$ and $(d)$ Axial velocity perturbation in a vertical plane corresponding to azimuth $\theta=0$. (c) Total velocity field, with the side cut of the primary spiralling vortices visible between $r=23.5$ and $r=25.5$. $(d)$ Azimuthal average of the axial velocity. In $(c)$ and $(d)$ the vertical scale has been multiplied by two to enhance visibility, and the domain has been truncated as no perturbations are detected upstream of $r=23.5$. On each view the left and right vertical lines mark the position of the primary and secondary front, respectively. 


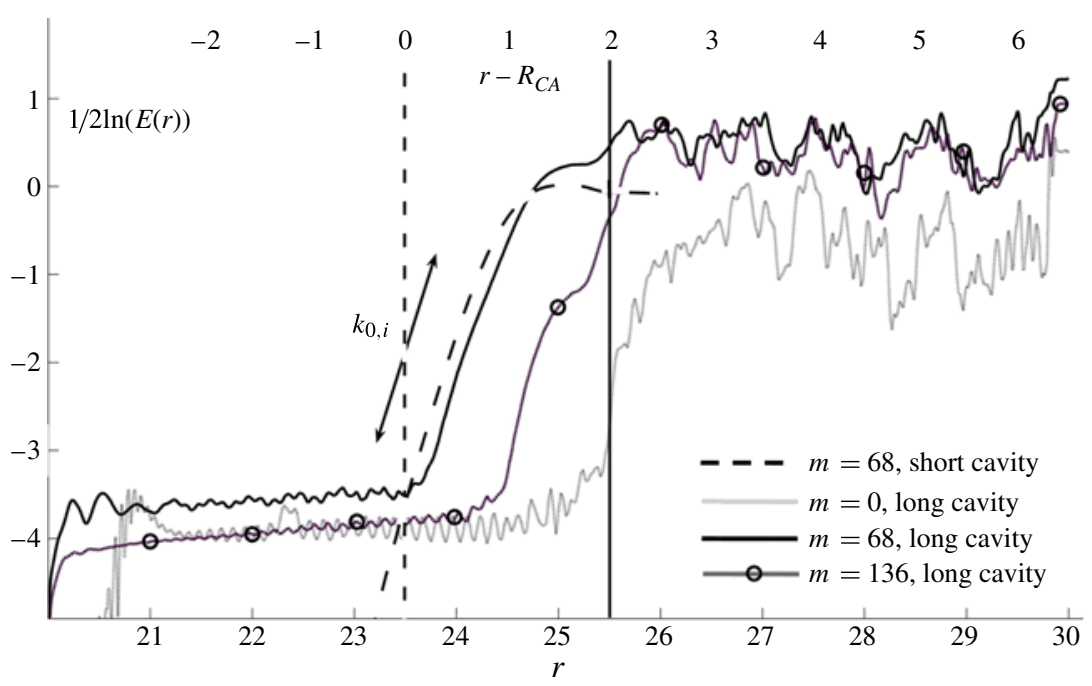

FIGURE 4. Linear-log amplitude of the perturbations of $m=68,0$ and 136 two fronts as a function of the radial coordinate, for $C_{w}=1995, \operatorname{Re}_{h}=780\left(\operatorname{Re}_{\delta} \in[330 ; 491]\right.$, $R o \in[-0.9 ;-0.45])$. The curve corresponding to $m=68$ in the short cavity has been plotted versus $r-R_{C A}$ for comparison since both simulations correspond to different flow parameters. The difference in saturation amplitude may be explained by the fact that the two fronts are obtained for different values of the Rossby number, $R o=0.81$ in the short cavity versus $R o=0.75$ in the longer one. The right vertical line marks the station at which the second front is visible on figure 3. The primary absolute spatial growth rate $k_{0, i}$ at the transition point is indicated by its slope. The curve for $m=0$ is extracted from the difference between the velocity fields at two times $(\delta t=0.018)$ to retain only the unsteady component and not the mean-flow modification.

existence of a subharmonic secondary instability mode of the cross-flow vortices over a single disc is not known for this particular Rossby number value.

To test the possibility of such a large-scale subharmonic mode, the computation is performed again in a $1 / 4$ sectorial cavity of same radial extent as mentioned in the numerics part. The initial perturbation is still a 68 -fold perturbation. Moreover the simulation in this $2 \pi / 4$ cavity was started again from $t=6$ adding at this time an impulsive noise exciting mode number 16. The dynamics in both cases gave a spatiotemporal diagram similar to figure 3(a), eventually leading to the same global mode structure as in the $1 / 68$ sectorial cavity with a first front leading to saturated cross-flow vortices followed downstream by a secondary front where transition to disordered state occurs. The only difference was that the disordered region progressively desynchronized from one 1/68 sector to the next resulting in an increase of the energy of all of the low wavenumbers. Shortly before blow-up and the end of the computation, the energy level associated with those low wavenumbers remains close to $10^{10}$ times weaker than the energy level of the mode numbers zero, 68, and its resolved harmonics. We conclude that the small-scale instability already observed in the 1/68 sector keeps being the mechanism for transition in larger sector. The growth of subharmonics occurs later as the result of the decorrelation of 1/68 sectors and not directly of subharmonic instability building on an unperturbed spiralling base flow as suggested for a single disc by Pier (2003). The instability does not involve Floquet azimuthal wavenumber close to 18 as predicted by Pier (2003) but Floquet number zero, the secondary instability having the same period as the primary flow. 


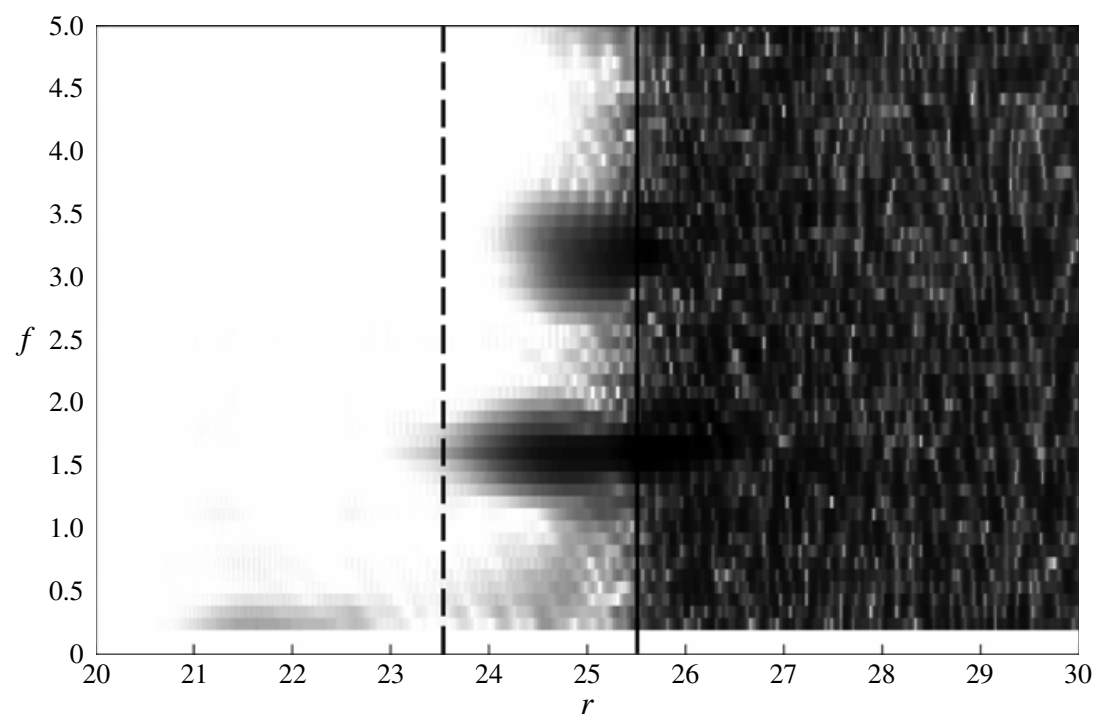

FIGURE 5. Time frequencies measured in the data presented on figure 3(a). At every radial station the energy spectrum is represented by mean of grey scale, low amplitudes are in white, large amplitudes are in black. Downstream of $r / h=25.5$ there is a sudden change in the spectrum. The left and right vertical lines mark the position of the primary and secondary front respectively, as extracted from figure 3 .

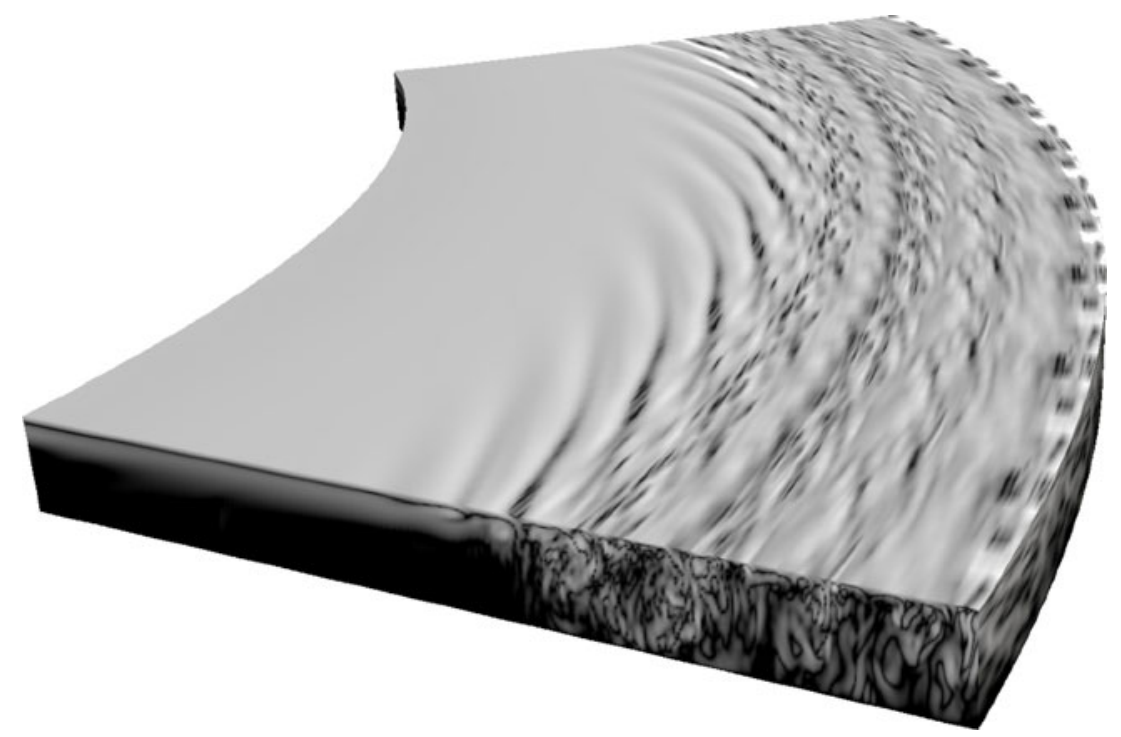

FIGURE 6 . Three-dimensional view of the vorticity field in the $2 \pi / 4$ cavity, obtained through DNS with the finer mesh shortly before blow-up.

\section{Discussion and conclusion}

The present results are fully consistent with the study in the short $(L=5)$ cavity of VSC where a steep-fronted nonlinear global mode, a so-called elephant mode, was observed. Figure 4 compares the present case with the prediction of the elephant-mode 
theory and with short cavity results. The total kinetic energy

$$
E(r)=r \int_{0}^{2 \pi} \int_{0}^{1 / 2} 1 / 2\left(u^{\prime 2}+v^{\prime 2}+w^{\prime 2}\right) \mathrm{d} z \mathrm{~d} \theta
$$

of the perturbation averaged in $z$ and $\theta$ presents a primary front located on the upstream limit of the absolutely unstable domain $r=R_{C A}$, followed downstream by a saturated wave and preceded upstream by a low-level perturbation region. The front location with respect to the predicted convective to absolute instability transition $R_{C A}$, its slope, the amplitude and starting point of the saturated wave agree with the elephant-mode prediction. In the present computations local analysis conducted using the DNS code yields a position $R_{C A}=23.5$ and a corresponding absolute spatial growth rate $k_{0, i}=3.61$, in excellent agreement with the location and the slope of the computed front measured on figure 4. As visible on figure $3(b)$ the wavelength shifts from $\lambda=30 \delta$ in the upstream part of the front $(r \in[23.5 ; 24.5])$ where amplitude is low to $\lambda=18 \delta$ in its downstream part $(r \in[24.5 ; 26])$ where the amplitude has saturated. These values agree with the absolute radial wavelength $\lambda=29 \delta$ predicted by the absolute instability analysis of Lingwood (1997) and by the presently computed value of $k_{0, r}$ (the absolute radial wavenumber) that should be valid ahead of the front where the amplitude is low and with $\lambda=20 \delta$, the wavelength of the saturated parallel wave beating at the global frequency $\omega_{g}$ according to Pier (2003). The spiralling arms corresponding to the saturated wave on figure $3(b)$ have a pitch of $\epsilon=31^{\circ}$, to be compared with the value of $\epsilon=30^{\circ}$ obtained by Pier (2003) for local nonlinear wave. Therefore, we can conclude that the upstream part of the nonlinear solution shown in figures 4 and $3(b)$ corresponds to an elephant mode due to the type I absolute instability as already described in VSC. Since in each cavity the mass-flow rate is chosen to place $R_{C A}$ at the position which optimizes the use of the computational domain: the flow conditions differ between the two simulations but comparison may still be achieved by plotting of the short cavity as a function of $r-R_{C A}$. In figure 4 the major difference between the short and the long cavities is that a secondary front follows the primary in the longer cavity, whereas it has no space to develop before reaching the outlet of the computational domain in the small cavity. It was noted in figure $3(a)$ that the primary and secondary instabilities exhibit fronts whose position is undergoing some small and slow vacillations. These may be accounted for at the beginning by the establishment time of the fronts, and next (after $t=8$ ) by variations in the high wavenumber dissipation. It is further argued that as the two curves in figure 2 cross at a very shallow angle (inducing a strong sensitivity to perturbation of the $R_{C A}$ location), the vacillation of the fronts may correspond to a small coupling between the fronts and the perturbation that changes the mean flow properties with a feedback on the $R_{C A}$ location of the mean flow.

In the long cavity the elephant mode is visible only for a short distance downstream, less than half the width of the primary front itself as seen on figure 4, since spatial and temporal disorder sets in at a secondary front located close to $r=25.5$, suggesting that the primary wave is already absolutely unstable to small-scale instability when it saturates.

Such a scenario of direct transition to disorder due to a cascade of absolute instabilities, the nonlinear wave resulting from the saturation of the primary instability being directly absolutely unstable to secondary instability, has been observed on the envelope equation (figure 12 of Couairon \& Chomaz 1999). The mode shape and the impulse response dynamics are in their case of amplitude equation strikingly similar to that reported here. This scenario, first proposed by Huerre (1988), was conjectured 
by Pier (2003) to explain the transition to turbulence over a rotating disc since, for Rossby number unity, he showed that the saturated cross-flow vortices beating at the global frequency are absolutely unstable, the subharmonic mode $m=18$ having the largest absolute growth rate. In the present double-facing-discs configuration, where the Rossby number is smaller than unity, the linear stability analysis of saturated crossflow vortices remains to be done. We presently observe the occurrence of secondary short-scale perturbations that reaches finite amplitude at the secondary front. This short-scale perturbations are present even whether a 68 -fold or a 4-fold symmetry is imposed, meaning that the Floquet azimuthal wavenumber of the perturbation is zero, and not subharmonic as predicted by Pier for the single disc. It is however presently unknown if this secondary instability is linear or nonlinear, i.e. linked to a linear or nonlinear absolute instability (Chomaz 1992). The difference in behaviour with that predicted by Pier (2003) may also be due to the interaction between the two crossflows over the facing discs. Indeed the difference with the single-disc configuration investigated in Pier (2003) lies in the effect of confinement on the secondary instability. Whereas former numerical study has shown that the primary instability is not affected by the presence of the second disc, it is clear from figures $3(c, d)$ and 6 that this may not hold for the secondary instability since perturbations fill the whole height of the computational domain after the secondary front.

To the best of the authors' knowledge the present study confirms for the first time the conjecture that transition to disorder, i.e. incipient turbulence, may take the form of an elephant-mode cascade with a primary front followed by a saturated wave already nonlinearly absolutely unstable and giving rise to a secondary front, a second-generation elephant mode living on the back of a primary elephant mode.

This work was granted access to the HPC resources of IDRIS under the allocation 2009-0242 made by GENCI (Grand Equipement National de Calcul Intensif).

\section{REFERENCES}

Akervik, E., Brandt, L., Henningson, D. S. \& Hoepffner, J. 2006 Steady solutions of the Navier-Stokes equations by selective frequency damping. Phys. Fluids 18, 126602.

Crespo del Arco, E., Serre, E., Bontoux, P. \& Launder, B. E. 2005 Stability, transition and turbulence in rotating cavities. In Advances in Fluid Mechanics (ed. M. Rahman). Dalhousie University Canada Series, vol. 41. pp. 141-196. WIT Press.

Chomaz, J. M. 1992 Absolute and convective instabilities in nonlinear system. Phys. Rev. Lett. 69, 1931-1934.

Chomaz, J. M., Huerre, P. \& Redekopp, L. G. 1991 A frequency selection criterion in spatially developping flows. Stud. Appl. Maths 84, 119-144.

Couniron, A. \& Chomaz, J. M. 1999 Primary and secondary nonlinear global instability. Physica D 132, 428-456.

DAVies, C. \& CARPenter, P. W. 2003 Global behaviour corresponding to the absolute instability of the rotating-disk boundary layer. J. Fluid Mech. 486, 287-329.

Davies, C., Thomas, C. \& CARpenter, P. W. 2007 Global stability of the rotating-disk boundary layer. J. Engng Maths 57, 219-236.

Gollub, J. P. \& Swinney, Harry L. 1975 Onset of turbulence in a rotating fluid. Phys. Rev. Lett. 35 (14), 927-930.

Hide, R. 1968 On source-sink flows stratified in a rotating annulus. J. Fluid Mech. 32, 737-764.

HUERRE, P. 1988 On the absolute/convective nature of primary and secondary instabilities. In Propagation in System Far from Equilibrium (ed. J. E. Wesfreid, H. R. Brand, P. Manneville, G. Albinet \& N. Boccara). pp. 340-353. Springer. 
LINGWOOD, R. J. 1995 Absolute instability of the boundary layer on a rotating disk. J. Fluid Mech. 299, 17-33.

LINGWOOD, R. J. 1996 An experimental study of the absolute instability of the rotating-disk boundary-layer flow. J. Fluid Mech. 314, 373-405.

LINGWOOD, R. J. 1997 Absolute instability of the Ekman layer and related rotating flows. J. Fluid Mech. 331, 405-428.

OWen, J. M. \& Pincombe, J. R. 1980 Velocity-measurements inside a rotating cylindrical cavity with a radial outflow of fluid. J. Fluid Mech. 99, 111-127.

OWen, J. M. \& Rogers, R. H. 1995 Heat Transfer in Rotating-disk System. Wiley.

Perret, G., Stegner, A., Dubos, T., Chomaz, J. M. \& Farge, M. 2006 Stability of parallel wake flows in quasigeostrophic and frontal regimes. Phys. Fluids 18, 126602.

PIER, B. 2003 Finite amplitude crossflow vortices, secondary instability and transition in the rotating-disk boundary layer. J. Fluid Mech. 487, 315-343.

Pier, B., Huerre, P., Chomaz, J. M. \& Couairon, A. 1998 Steep nonlinear global modes in spatially developing media. Phys. Fluids 10, 2433-2435.

Serre, E., Hugues, S., Crespo del Arco, E., Randriamampianina, A. \& Bontoux, P. 2001 Axisymmetric and three-dimensional instabilities in an Ekman boundary-layer flow. Intl J. Heat. Transfer Fluid Flow 22, 82-93.

SEVERAC, E. \& SERRE, E. 2007 A spectral vanishing viscosity for the les of turbulent flows within rotating cavities. J. Comput. Phys. 226, 1234-1255.

Tobias, S. M., Proctor, M. R. E. \& KNobloch, E. 1998 Convective and absolute instabilities of fluid flows in finite geometry. Physica D 113, 43-72.

Viaud, B., Serre, E. \& Chomaz, J. M. 2008 The elephant mode between two rotating disks. J. Fluid Mech. 598, 451-464.

Zandbergen, P. J. \& Dijkstra, D. 1986 Vonkármán swirling flows. Annu. Rev. Fluid Mech. 19, $465-491$. 